\title{
Propuesta didáctica en la formación de profesorado para trabajar naturaleza de la ciencia y pensamiento crítico
}

\author{
Cristina Cobo Huesa, Ana María Abril Gallego, Marta Romero Ariza \\ Departamento de Didáctica de las Ciencias. Facultad de Humanidades y Ciencias de la \\ Educación. Universidad de Jaén (España).
}

[Recibido el 28 de octubre de 2018, aceptado el 18 de febrero de 2019]

\begin{abstract}
La demanda social de educar a ciudadanos alfabetizados científicamente convierte en prioritario formar a profesorado con una adecuada comprensión sobre la naturaleza de la ciencia (NDC) y desarrollo de pensamiento crítico $(P C)$, requisitos indispensables para poder impartir dichos contenidos en el aula. Para tal fin, en este trabajo, se presenta el diseño de una propuesta didáctica (prototipo), que está siendo evaluada y mejorada a través de la metodología de investigación enfocada al diseño. Este prototipo integra la indagación y la historia de la ciencia a través de la controversia científica de la generación espontánea, dentro de un marco explícito-reflexivo. Con el fin de mejorar su diseño y eficacia, se están llevando a cabo ciclos iterativos de pilotaje que, hasta el momento, han mostrado la importancia de adoptar un enfoque guiado en las tareas de indagación y de un enfoque explícito en la instrucción sobre la NDC y el PC para lograr un aprendizaje exitoso y significativo.
\end{abstract}

Palabras clave: pensamiento crítico, DBR; historia de la ciencia; indagación; naturaleza de la ciencia.

\section{Didactical approach in the teacher training to work with nature of science and critical thinking}

The social demand to educate scientifically literate citizens becomes crucial to train teachers with an adequate understanding of nature of science (NOS) and development of critical thinking (CT), both essential requirements to instruct these contents in classroom. Because of this aim, in this work, we present the design of a didactical approach (prototype), which is being evaluated and improved through the Design-Based Research methodology. This prototype integrates the inquiry and the history of science through the scientific controversy of spontaneous generation, within an explicit-reflective framework. In order to improve the design and efficacy of the prototype, we are carrying out iterative piloting cycles which, so far, have revealed the importance of adopting an oriented approach in inquiry tasks and an explicit approach in NOS and CT instruction to achieve a successful and significant learning.

Keywords: critical thinking; DBR; history of science; inquiry; nature of science.

Para citar el artículo. Cobo-Huesa, C., Abril, A.M. y Ariza, M.R. (2019). Propuesta didáctica en la formación de profesorado para trabajar naturaleza de la ciencia y pensamiento crítico. Ápice. Revista de Educación Científica, 3(1), 15-28. DOI: https://doi.org/10.17979/arec.2019.3.1.4630

Contacto.cchuesa@ujaen.es, amabril@ujaen.es, mromero@ujaen.es 


\section{Introducción}

La necesidad de mejorar la alfabetización científica de los ciudadanos queda reflejada en el actual dominio de la ciencia y la tecnología en todos los aspectos de nuestra vida, considerándose el pilar fundamental de la educación científica (OCDE, 2017). El Programa Internacional para la Evaluación de Estudiantes (PISA) 2015, la define como la habilidad de participar en temas relacionados con la ciencia y la tecnología como ciudadanos reflexivos (OCDE, 2017). Este informe sugiere un marco de competencias relacionadas con el desarrollo de habilidades científicas (explicar fenómenos científicamente, evaluar y diseñar investigaciones científicas e interpretar datos y pruebas de manera científica), que requiere, no solo un conocimiento sobre contenidos disciplinares y procedimentales de la ciencia, sino también un conocimiento sobre las características del conocimiento científico y los procesos implicados en su construcción.

La escuela debe legitimar su papel si quiere contribuir al empoderamiento de las nuevas generaciones con el conocimiento, habilidades de pensamiento y actitudes necesarios para participar democráticamente en los asuntos científicos de relevancia social. Por ello, este estudio tiene como objetivo mejorar la formación inicial del profesorado, incidiendo explícitamente en la mejora de su comprensión sobre la naturaleza de la ciencia (NDC) y su pensamiento crítico (PC), dos elementos indispensables para promover una educación científica de calidad, a través del diseño de una intervención basada en la investigación y la literatura especializada.

\section{La naturaleza de la ciencia en la enseñanza de las ciencias}

Una alfabetización científica holística implica la adecuada comprensión sobre qué es la ciencia y cómo funciona el entramado de la empresa científica, conocimiento conocido como NDC. La perspectiva que ha prevalecido en la literatura internacional sobre la NDC refleja una visión consenso reduccionista enfocada únicamente a aspectos epistemológicos (Lederman, Abd-El-Khalick, Bell y Schwartz, 2002). Este enfoque resalta las características del conocimiento científico (naturaleza empírica de la ciencia, diferencia entre teorías y leyes científicas, carácter tentativo, creativo y carga teórica del conocimiento científico) y de los procesos de la ciencia (observaciones, inferencias y metodologías científicas). De modo que, los aspectos sociológicos, relacionados con la construcción y validación social del conocimiento científico, así como con las complejas relaciones que se establecen entre la ciencia, la tecnología y la sociedad, quedan reflejados en una dimensión denominada integración social y cultural del conocimiento científico.

Este enfoque ha sido criticado por autores que consideran que la NDC debe ofrecer una visión más amplia y funcional de la ciencia, que atienda plenamente al contexto y demanda de la sociedad, abogando por incluir la enseñanza de la NDC en el marco CienciaTecnología-Sociedad (CTS) (Acevedo-Díaz y García-Carmona, 2016; Vázquez-Alonso y Manassero-Mas, 2013).

En el marco CTS, además de incluir rasgos epistemológicos de la ciencia, cobran vital importancia las normas y los valores que rigen la comunidad científica, las características de los científicos y las relaciones que se establecen en el complejo sistema CTS, ofreciendo una visión holística, realista y funcional sobre cómo funciona la ciencia y de qué manera influimos y somos influenciados por los avances científico-tecnológicos. De acuerdo a este enfoque, se ha desarrollado empíricamente el Cuestionario de Opiniones sobre Ciencia, Tecnología y Sociedad (COCTS), con el fin de favorecer el desarrollo y evaluación de estrategias didácticas enfocadas a mejorar la concepción que los individuos tienen sobre la NDC (Manassero, Vázquez y Acevedo, 2001). Por ello, consideramos que la enseñanza de 
la NDC bajo el enfoque CTS es el más pragmático, tanto desde el punto de vista formativo de los individuos como de evaluación de la eficacia de estrategias de instrucción diseñadas para mejorar la comprensión de la NDC.

Junto a todos estos aspectos, el enfoque CTS contribuye a que la enseñanza de la NDC se presente como un elemento crucial para la educación científica de los individuos. Conocer acerca de los procesos de construcción y validación del conocimiento científico les capacita para emitir juicios coherentes a su fiabilidad y limitaciones de manera informada, contribuye a valorar el esfuerzo humano en comprender los fenómenos de la naturaleza a lo largo de los años y, en consecuencia, a considerar la ciencia como un elemento esencial de nuestra cultura y capaz de ayudarnos a hacer frente a las actuales problemáticas de la humanidad (sanidad, agotamiento de recursos, cambio climático, etc.). En esta línea, también ayuda a conocer las normas y valores morales que rigen la comunidad científica, necesarios para que los procesos de la ciencia estén acordes a las necesidades de la sociedad (Driver, Learch, Millar y Scott, 1996).

Finalmente, cabe resaltar que una adecuada comprensión de la NDC facilita el aprendizaje de la misma ciencia, mejorando la asimilación de conceptos e ideas científicas (Driver et al., 1996; Moreno, Zúñiga, Cofré y Merino, 2018), así como el desarrollo de destrezas del PC y viceversa (Vázquez-Alonso y Manassero-Mas, 2018).

\section{El pensamiento crítico en la educación científica}

Conseguir que los individuos logren aplicar adecuadamente el conocimiento científico en contextos CTS implica ir más allá de la comprensión sobre la ciencia. Para ello, es necesario dotar a los individuos de las herramientas intelectuales necesarias para evaluar el conocimiento científico y el mundo que les rodea, contribuyendo a su emancipación social en la toma de decisiones sobre asuntos científicos y socio-científicos. En consecuencia, la enseñanza del PC se presenta como un componente clave en la educación científica (Jiménez-Aleixandre y Puig, 2012).

Existen múltiples definiciones para el PC, pero, de todas ellas, se deduce que se trata de una habilidad cognitiva de orden superior que puede definirse como un proceso metacognitivo, razonado, reflexivo y consciente, que integra diversas destrezas, con el fin de mejorar nuestro propio pensamiento y lograr nuestras metas (Ennis, 1991; Facione, 2011; Halpern, 2014; Zoller y Nahum, 2012). Aunque existe el consenso de que el PC está integrado por un componente cognitivo y otro disposicional, no hay unanimidad respecto a las destrezas y disposiciones que lo integran.

Respecto al componente cognitivo, Halpern (2014) estableció cinco dimensiones amplias de PC: la comprobación de hipótesis, el razonamiento verbal, el análisis de argumentos, la resolución de problemas y toma de decisiones y, por último, el pensamiento implicado en situaciones de probabilidad e incertidumbre. Estas dimensiones, a su vez, son desglosadas en una serie de destrezas. En concreto, la dimensión de comprobación de hipótesis implica comprender la necesidad de controlar y aislar variables, o identificar las relaciones entre dos o más variables, distinguiendo entre causalidad y correlación. Por su parte, la dimensión de razonamiento verbal, relacionada con la defensa frente a las técnicas persuasivas del lenguaje cotidiano, implica reconocer la ambigüedad en los argumentos o el análisis de analogías.

En consecuencia, podemos apreciar que la taxonomía propuesta por Halpern recoge de manera reducida y operativa las grandes destrezas del PC, agrupadas en dimensiones. Además, el instrumento de evaluación desarrollado en base a esta taxonomía (Halpern, 
2010) se presenta como un medio interesante para valorar la transferencia de estas destrezas a contextos diferentes de los meramente educativos, ya que evalúa las destrezas del PC a través de situaciones contextualizadas en temas cotidianos similares a los encontradas en la vida real.

En cuanto al componente disposicional, existe el consenso de que, sin una predisposición a emplear el PC, no se aplican las destrezas, aunque estén bien consolidadas en los individuos. Un estudio llevado a cabo por Valenzuela, Nieto y Muñoz (2014) sugiere que la variabilidad en el desempeño del PC se explica mejor por la motivación que por las disposiciones, entendidas estas últimas como actitudes o hábitos intelectuales consolidados (Ennis, 1991; Facione, 2011; Halpern, 2014), defendiendo que el respaldo teórico del enfoque motivacional, basado en el modelo de expectativa/valor, es mucho mayor al de las disposiciones.

En esta línea, con el fin de superar las dificultades en la poca consensuada dimensión disposicional, Valenzuela (2007) desarrolló una escala para valorar la motivación a emplear el PC, teniendo en cuenta las expectativas que tienen los individuos de emplear el PC y el valor que le asignan a su empleo de acuerdo a la importancia de logro, interés, utilidad y costo.

\section{Ideas del profesorado en formación inicial sobre la NDC y el PC}

Como hemos visto, los constructos de NDC y PC son complejos y cambiantes, lo que dificulta su comprensión e implementación eficaz en el aula, convirtiéndose en todo un reto docente.

Por un lado, el profesorado en formación inicial suele presentar la concepción de que el conocimiento científico es estático, verdadero y absoluto, confunde observación e inferencia, asume la objetividad de los científicos y que estos se limitan a aplicar el método científico y a elaborar leyes y teorías científicas en base al registro de hechos sin recurrir a la creatividad. Además, cree en una jerarquía hipótesis-teorías-leyes, no tienen una concepción clara sobre las motivaciones de los científicos, sus valores y estándares, y presentan un conocimiento poco concreto sobre las relaciones CTS (Demirdöğen, Hanuscin, Uzuntiryaki-Kondakci y Köseoğlu, 2016; Irez, 2006; Vázquez-Alonso y Manassero-Mas, 2013). En consecuencia, mejorando la concepción del profesorado sobre la NDC se espera contribuir a la incorporación de estos aspectos en sus instrucciones de aula e influir en la forma y contenido de su enseñanza, coherente a los rasgos de la NDC.

Por otro lado, la revisión de la literatura muestra que el profesorado en formación inicial presenta un bajo dominio de las destrezas del PC (Qing, Jing y Yan, 2010), una disposición media-baja a emplearlo (Akgun y Duruk, 2016; Dermihan y Köklükaya, 2014) y considera su empleo como una actividad costosa (Valenzuela y Saiz, 2010), lo que dificulta aún más su desarrollo. Además, gran parte del profesorado en formación considera que la enseñanza del PC es más propia de las asignaturas pedagógicas (Solbes-Matarredona y TorresMerchán, 2013), lo que sugiere la necesidad de articular el PC con las disciplinas científicas a través de un cambio metodológico en las aulas.

Todos estos aspectos ponen de manifiesto la importancia de desarrollar estrategias de instrucción enfocadas a mejorar la comprensión de la NDC y el PC del profesorado desde su formación inicial, haciéndole partícipe de propuestas didácticas que puedan constituir un referente para que sus futuras prácticas educativas ofrezcan una educación científica que responda de manera funcional a por qué y para qué enseñar ciencias. 


\section{Pregunta y objetivos de investigación}

Con el fin de contribuir a una educación científica de calidad que atienda a las necesidades de la sociedad actual, resulta fundamental focalizar nuestros esfuerzos en promover la comprensión sobre la NDC y el PC del profesorado en formación inicial. En consecuencia, nuestra pregunta de investigación es la siguiente: ¿Qué características debe tener una instrucción eficaz enfocada a mejorar la comprensión de la NDC y el PC del profesorado en formación inicial?

Teniendo en cuenta lo anteriormente expuesto, los objetivos de este trabajo son:

- Diseñar una secuencia de enseñanza-aprendizaje (SEA) basada en la investigación especializada que integre elementos de probada utilidad para promover la NDC y el PC en profesorado en formación inicial.

- Pilotar y mejorar la SEA inicial (prototipo) atendiendo a los resultados de la evaluación sistemática de la implementación.

\section{Metodología}

Para diseñar la propuesta didáctica se empleó la metodología de investigación enfocada al diseño o DBR ("Design Based Research"). Esta estructura de investigación se caracteriza por la generación de productos (en este caso una SEA) y la obtención de principios de diseño basados en la investigación y evaluación sistemática (Romero-Ariza, 2014).

La metodología DBR se estructura en tres fases: una primera fase de investigación enfocada a revisar la literatura especializada con el fin de establecer el marco teórico de la investigación; una segunda fase de desarrollo y pilotaje, que engloba el diseño del prototipo y su mejora progresiva a través de sucesivos ciclos de aplicación y evaluación; y una tercera fase de evaluación final, en la que se valora si la propuesta final satisface los objetivos iniciales a través del análisis y la reflexión sobre las características que han asegurado su efectividad.

En este tipo de investigación se produce una retroalimentación entre teoría y práctica, permitiendo disminuir la brecha existente entre la investigación y la práctica educativa, dotando de mayor relevancia y utilidad a la investigación especializada para resolver problemas educativos en contextos reales.

En el presente estudio, como parte de la primera fase de investigación, se llevó a cabo una revisión sistemática de la literatura para identificar las características que debía presentar una intervención eficaz para alcanzar nuestro objetivo. Para ello, se realizó la búsqueda en la literatura de artículos basados en el análisis de intervenciones para mejorar la comprensión de la NDC y el PC, así como de revisiones bibliográficas sobre la enseñanza-aprendizaje de ambos constructos, con el fin de identificar pautas de instrucción que pudieran emplearse para su enseñanza conjunta. Tras ello, para asegurar la coherencia del diseño de la intervención y nuestro objetivo, el equipo de investigación analizó los principios de diseño extraídos de la revisión bibliográfica y llegó a un acuerdo consensuado sobre la mejor estrategia de instrucción.

Para evaluar la efectividad del prototipo, se empleó un diseño de investigación pre-post test cuantitativo, a través de la aplicación de instrumentos validados y previamente empleados en otros estudios (Halpern, 2010; Manassero et al., 2001; Valenzuela, 2007), combinado con el estudio cualitativo de casos a través del análisis de las producciones escritas de los estudiantes, que incluye la categorización y el análisis de contenido, para poder triangular los resultados con el análisis pre-post test. 


\section{Resultados}

\section{Investigación preliminar}

De acuerdo a la literatura, existe el consenso de que las estrategias de instrucción sobre NDC y PC han de ser explícitas y reflexivas (Khishfe y Abd-El-Khalick, 2002; Marin y Halpern, 2011). Dentro de este enfoque, la dimensión reflexiva requiere una alta demanda cognitiva, surgiendo una convergencia entre la enseñanza de la NDC y el PC (Vázquez-Alonso y Manassero-Mas, 2018; Yacoubian, 2015).

Los métodos más efectivos para el aprendizaje de la NDC y el PC son aquellos que implican el aprendizaje activo en contextos auténticos que dotan de significado las tareas a desarrollar (Abrami et al., 2015; Blanco-López, España-Ramos y Franco-Mariscal, 2017; Yacoubian, 2015). Dentro de este enfoque pedagógico, encontramos el aprendizaje por indagación, que implica al alumnado en los procesos científicos, promoviendo el empleo del PC, habilidades científicas y contribuyendo a la mejora de la comprensión de la NDC, a través de un enfoque explícito y reflexivo (Yacoubian y BouJaoude, 2010). Además, a través de la indagación guiada, en la que el docente interacciona con el alumnado, se obtienen mejores resultados de aprendizaje (Abrami et al., 2015).

Del mismo modo, uno de los recursos más empleados para contextualizar la enseñanza de la NDC es la historia de la ciencia (HDC), ya que ilustra cómo funciona la comunidad científica y las interacciones CTS (McComas, 2011). Acevedo-Díaz, García-Carmona y Aragón (2017) proponen utilizar la HDC mediante el análisis crítico de controversias científicas, al suponer un medio idóneo para la reflexión y el diálogo, y resaltan una serie de aspectos, como incluir textos originales y mostrar los errores del proceder científico, para evitar dar una imagen deformada de la ciencia.

No obstante, McComas (2011) destaca la necesidad de enfocar la HDC de manera que motive y comprometa al alumnado, aspecto necesario no solo para mejorar su comprensión sobre la ciencia, sino también para promover el empleo del PC. Para tal fin, la indagación científica podría presentarse como un elemento dinamizador que reformule el empleo de la HDC y, al mismo tiempo, contribuya a la comprensión de la NDC y al desarrollo del PC.

\section{Diseño del prototipo}

En base a la investigación preliminar expuesta, se llevó a cabo el diseño del prototipo inicial de nuestra propuesta didáctica, denominado "Investigando sobre el origen de los seres vivos", contextualizado en la controversia científica de la generación espontánea (GE) (Figura 1).

La elección de esta controversia está fundamentada por diversas razones:

- Ilustra aspectos epistemológicos y sociológicos de la NDC, aportando una visión global acerca del funcionamiento de la ciencia y los factores internos y externos a la comunidad científica que influyen en la construcción del conocimiento científico.

- Al tratarse de una controversia, permite el análisis de diferentes posturas, afirmaciones científicas y diseños experimentales, lo cual supone un ambiente idóneo para el desarrollo de habilidades y actitudes críticas.

- La controversia de la GE permite establecer conexiones entre las experiencias del alumnado y las teorías científicas, creando situaciones que provoquen un conflicto cognitivo que le motive a participar en la tarea, contribuyendo positivamente en el componente motivacional necesario para entrenar y aplicar el PC. 
PROTOTIPO PARA PROMOVER LA COMPRENSIÓN DE LA NDC Y EL PC INVESTIGANDO SOBRE EL ORIGEN DE LOS SERES VIVOS

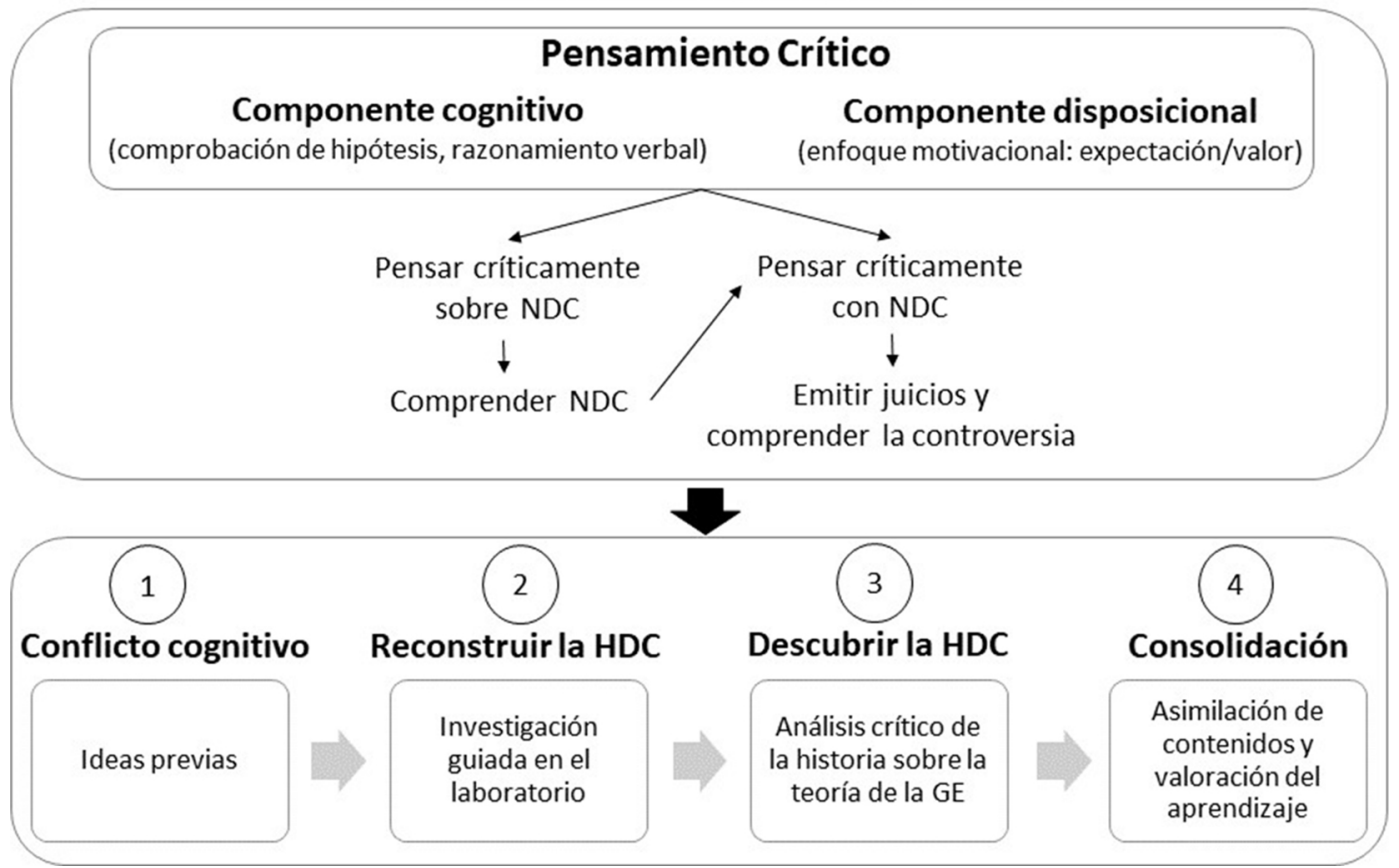

Figura 1. Esquema de la fundamentación y estructura del diseño del prototipo "Investigando sobre el origen de los seres vivos"

El prototipo diseñado integra el aprendizaje por indagación a través del análisis crítico de la HDC y la participación activa del alumnado en escenarios de aprendizaje que permiten reconstruirla. Como aparece reflejado en la Figura 1, y tomando como referencia el marco teórico propuesto por Yacoubian (2015), las actividades planteadas en el prototipo tienen como finalidad que el alumnado piense críticamente sobre algunos rasgos de la NDC para favorecer su comprensión, y posteriormente, aplique ese conocimiento adquirido o refinado sobre la NDC para comprender la controversia y emitir juicios fundamentados sobre ella. Concretamente, las actividades propuestas implican el empleo de las destrezas cognitivas de comprobación de hipótesis y razonamiento verbal, pues el alumnado se implica en el diseño de un experimento para comprobar sus hipótesis sobre el origen de los seres vivos, en la evaluación de los diseños experimentales de algunos de los científicos más relevantes de la época, así como en la valoración de la calidad de diferentes ideas y razonamientos en torno a la controversia de la GE, como se detallará en la descripción de las fases del prototipo. Del mismo modo, con este diseño se pretende contribuir al aspecto motivacional del empleo del PC, no solo mostrando la importancia y valor de su empleo para el adecuado desempeño de las tareas propuestas, sino también visibilizando cómo el cuestionamiento de las teorías científicas imperantes y la continua revisión del conocimiento científico permiten el avance de la ciencia.

A continuación, se describe con más detalle cada una de las fases del prototipo:

1. Conflicto cognitivo: a través de un pequeño fragmento sobre Aristóteles, uno de los primeros defensores de la GE, se pretende introducir la temática de la propuesta didáctica y evidenciar las ideas previas del alumnado sobre el origen de los seres vivos y algunos rasgos de la NDC.

2. Reconstruir la HDC: de manera previa al descubrimiento de la HDC, a través de un escenario de aprendizaje basado en la indagación guiada en el laboratorio, el alumnado 
tendrá que reconstruir la HDC, es decir, intentar dar una explicación científica al origen de los seres vivos, al igual que hicieron los científicos de la época.

Con el fin de procurar contextos significativos que impliquen cognitiva y emocionalmente al alumnado, esta tarea se contextualiza en la elaboración de una explicación acerca de la aparición de moho en la fruta que refute la teoría de la GE. Para ello, el alumnado se implicará en la formulación de hipótesis, el diseño experimental, el análisis de resultados y la extracción de conclusiones a través de un razonamiento lógico basado en las pruebas recogidas, incidiendo en el desarrollo de las destrezas del PC indicadas.

Una vez finalizada la tarea de indagación, para explicitar los rasgos sobre la NDC trabajados durante su desarrollo (subjetividad de la práctica científica, provisionalidad del conocimiento científico, aceptación de teorías científicas y papel de los errores en la ciencia), el alumnado reflexionará sobre ellos a través de una serie de cuestiones. Por ejemplo, para promover la reflexión acerca de la subjetividad de la práctica científica se formularon las siguientes preguntas:

"De manera previa a la experimentación, ¿tus compañeros y tú disteis, inicialmente, la misma explicación sobre la aparición de moho en la naranja?"

“¿Creéis que diferentes científicos/as competentes explican las observaciones sobre los fenómenos naturales de la misma manera?"

3. Descubrir la HDC: el alumnado continuará con la tarea de indagación a través del análisis crítico de las declaraciones y los experimentos realizados por algunos de los científicos más relevantes implicados en la controversia de la GE. Esta tarea incluye fragmentos de texto adaptados, uno de ellos extraído de una obra original, y una serie de cuestiones asociadas a ellos que conlleva la reflexión sobre la NDC y el PC.

Tabla 1. Actividad extraída del prototipo en la que se muestra la fundamentación teórica de la propuesta didáctica "Investigando sobre el origen de los seres vivos"

\begin{tabular}{|l|l|l|}
\hline Tarea cognitiva & Actividad & Propósito didáctico \\
\hline $\begin{array}{l}\text { Pensar } \\
\text { críticamente } \\
\text { sobre NDC }\end{array}$ & $\begin{array}{l}\text { Reflexionad sobre el contenido y el } \\
\text { significado de las siguientes frases, } \\
\text { extraídas de la obra "Ortus Medicinae" } \\
\text { de Jean Baptiste van Helmont para } \\
\text { decidir si es una observación o una } \\
\text { inferencia: } \\
\text { a. “El fermento, surgiendo de la ropa } \\
\text { interior y penetrando a través de las } \\
\text { cáscaras de trigo, cambia el trigo en } \\
\text { ratones" } \\
\text { b. "Al cabo de 21 días, aparecen ratones } \\
\text { entre la ropa sucia y el trigo" }\end{array}$ & $\begin{array}{l}\text { Aplicar el PC para clasificar es } \\
\text { frases, con de diferenciar } \\
\text { inferencia (frase a), contribuyendo } \\
\text { a diferenciar ambos procesos } \\
\text { científicos y a mejorar la } \\
\text { comprensión del alumnado sobre } \\
\text { la subjetividad de la práctica } \\
\text { científica. }\end{array}$ \\
\hline $\begin{array}{l}\text { Pensar } \\
\text { críticamente } \\
\text { con NDC }\end{array}$ & $\begin{array}{l}\text { ¿Por qué Jean Baptiste van Helmont } \\
\text { realizaba inferencias a favor de la GE? }\end{array}$ & $\begin{array}{l}\text { Emitir juicios sobre la controversia } \\
\text { tras comprender el papel de las } \\
\text { creencias de los científicos en } \\
\text { el diseño de sus investigaciones } \\
\text { y la aceptación de las teorías } \\
\text { científicas. }\end{array}$ \\
\hline
\end{tabular}

Con ello, se pretende que el alumnado reflexione sobre el origen de la controversia, mostrándole cómo la propia naturaleza de los procesos científicos y las características de los 
científicos (motivaciones, valores y estándares) influyen en la aceptación/rechazo de una teoría científica, dando lugar a una controversia entre la comunidad científica que, en última instancia, también influye en la concepción que la sociedad tiene sobre los fenómenos naturales.

En la Tabla 1, se muestra una actividad extraída de esta fase del prototipo. En ella, el alumnado tendrá que distinguir entre las observaciones y las inferencias realizadas por Jean Baptiste van Helmont, conocido por desarrollar un experimento en el que, al igual que un libro de recetas, sugería el origen de los ratones a partir de trigo y ropa sucia. El alumnado tendrá que reflexionar sobre los resultados extraídos de este experimento y sobre la coherencia de los mismos y la conclusión extraída, evaluando, así, la validez del razonamiento empleado por el investigador en sus inferencias.

4. Consolidación: a través de una serie de cuestiones de reflexión, se incidirá, de manera explícita, en la asimilación de los contenidos sobre NDC trabajados, que evidenciarán la comprensión del alumnado sobre la controversia científica. También permitirán valorar la importancia que el profesorado en formación inicial otorga al desarrollo de la comprensión de la NDC y el PC en la enseñanza de las ciencias, así como al empleo de la metodología de indagación y el recurso de la HDC para dicho fin.

\section{Ciclos de pilotaje y mejora del prototipo}

Atendiendo a la estructura de investigación DBR, para evaluar la efectividad del prototipo diseñado, se están llevando a cabo ciclos de aplicación, evaluación y mejora con los que refinar su diseño hasta lograr su optimización.

Hasta ahora, se ha realizado un primer ciclo de refinamiento del diseño inicial del prototipo con un pequeño grupo de estudiantes de un curso de profesorado en formación inicial del Máster Universitario en Profesorado de Educación Secundaria. Posteriormente, realizamos evaluación formal del diseño del prototipo según las observaciones recogidas en el aula, tras la cual, llevamos a cabo un segundo ciclo de refinamiento del prototipo mejorado con un grupo mayor de profesorado en formación inicial del Grado de Educación Primaria.

Para evaluar la efectividad del prototipo, desde el punto de vista cuantitativo, antes y después de su implementación, se han empleado tres instrumentos. Por un lado, para valorar las creencias del profesorado en formación inicial sobre la NDC se han empleado las cuestiones 10113, 20821, 60111, 60211, 70211, 70221, 70411, 90111, 90411 y 90651 del COCTS en su modelo de respuesta múltiple (Manassero et al., 2001). Por otro lado, para la evaluación del PC se ha empleado la forma corta de reconocimiento del cuestionario de evaluación del pensamiento mediante situaciones cotidianas de Halpern (2010), concretamente las 10 situaciones sobre comprobación de hipótesis y razonamiento verbal. Por último, se ha utilizado la escala motivacional de PC desarrollada por Valenzuela (2007).

Desde un punto de vista cualitativo, se está procediendo al análisis de las producciones de los participantes, categorizando y realizando el análisis de contenido para poder triangular los resultados con el análisis pre-post test.

\section{Primer ciclo de refinamiento}

Inicialmente, el prototipo fue implementado en un grupo de 28 estudiantes del Máster Universitario en Profesorado de Educación Secundaria. Debido a las limitaciones temporales, no pudo desarrollarse la indagación guiada en el laboratorio, por lo que esta primera implementación se enfocó, principalmente, a analizar el diseño y efectividad de la fase de descubrimiento de la HDC. 
Dado que los productos y datos recogidos se encuentran en fase de procesamiento y análisis, la evaluación del prototipo se llevó a cabo, en primera instancia, a través de las observaciones recogidas en el aula. Esta primera experiencia evidenció que el alumnado se mostró implicado en el desempeño de la tarea, aunque parte de él percibió demasiado extensa la actividad y dudaba sobre el propósito de algunas cuestiones.

Por tanto, este ciclo de refinamiento fundamentalmente permitió realizar una serie de mejoras formales, simplificando la estructura de la tarea y reformulando algunas cuestiones para enfocarlas de manera efectiva a la consecución de los objetivos planteados. Para ilustrar alguna de estas modificaciones, en el prototipo, tras analizar el experimento de Jean Baptiste van Helmont, se expone al alumnado el experimento que Francisco Redi diseñó para refutar la aparición de gusanos a partir de carne en descomposición. En este contexto, se presenta un argumento que defiende la refutación de la teoría de la GE debido a los resultados obtenidos por este investigador y se le pide al alumnado que aporte pruebas a favor y en contra de dicha afirmación. Esta cuestión tenía la intención de que el alumnado resaltara, como prueba a favor, la mayor rigurosidad del experimento de este investigador para comprobar su hipótesis, y como prueba en contra, que cuestionara el razonamiento del argumento presentado, valorando que para refutar una teoría científica es necesario que la nueva teoría sea comprobada con éxito en varias ocasiones y contextos. No obstante, el alumnado reconoció no saber contestar, necesitando que la docente explicitara los aspectos que debían tener en cuenta en relación al diseño del experimento, sus resultados y la conclusión presentada.

Así pues, para mejorar el prototipo, el equipo investigador decidió modificar esta cuestión con el fin de que el alumnado reflexionara explícitamente sobre los aspectos indicados. Por ello, presentando el mismo argumento, se decidió cambiar la pregunta haciéndoles reflexionar sobre su grado de acuerdo con dicha afirmación, justificando su respuesta en base al diseño y los resultados obtenidos por el investigador.

\section{Segundo ciclo de refinamiento}

Una vez mejorado el prototipo, se volvió a implementar en un curso de 98 estudiantes del Grado en Educación Primaria. En este caso, sí pudo implementarse el prototipo completo.

Al igual que en el primer ciclo de refinamiento, realizamos una evaluación preliminar del diseño del prototipo mejorado basada en las percepciones del aula. El alumnado mostraba interés, participación e implicación en el desarrollo de las tareas. No obstante, esta implementación permitió detectar que, en su mayoría, el alumnado tenía dificultad para desarrollar la tarea de indagación, necesitando de la continua interacción con la docente para iniciar el diseño de sus experimentos. Respecto a la tarea de análisis de la HDC, se percibió que el alumnado se desenvolvía mejor en la resolución de las cuestiones. Sin embargo, la docente detectó que en preguntas que hacían alusión a aspectos sociológicos de la construcción del conocimiento científico, el alumnado carecía del conocimiento necesario para contestar de manera fundamentada. Así, en cuestiones como la mostrada en la Tabla 1, era conveniente que el alumnado dispusiese de información acerca del contexto histórico en el que Jean Baptiste van Helmont desarrolló su investigación para justificar de manera informada cómo las creencias del investigador pudieron influir en la realización de inferencias a favor de la GE.

Por ello, el equipo investigador ha decidido incorporar al prototipo una serie de preguntas de metacognición, previas al diseño experimental, con el fin de que el alumnado reflexione explícitamente sobre los aspectos necesarios para llevar a cabo un diseño experimental riguroso y tome control de sus acciones. Igualmente, se ha incorporado una breve descripción acerca del contexto social que caracteriza la época en la que vivieron los investigado- 
res implicados en la controversia de la GE y se ha destacado la formación de los mismos, para que el alumnado pueda construir un aprendizaje más significativo.

\section{Discusión y consideraciones finales}

Con el prototipo de SEA propuesto, se pretende mejorar la comprensión sobre la NDC y el PC del profesorado en formación inicial, dada la importancia de ambos elementos para conseguir una educación científica que permita a los individuos desenvolverse con eficacia y autonomía en un mundo dominado por la ciencia y la tecnología (OCDE, 2017).

En este trabajo, se ha mostrado el diseño, evaluación preliminar y mejora del prototipo inicial de la SEA, enmarcado en la estructura de investigación DBR. Esta metodología, basada en la investigación especializada y en ciclos iterativos de pilotaje y desarrollo, está permitiendo su refinamiento y optimización. Actualmente, la investigación se encuentra en la fase de procesamiento y análisis de datos. No obstante, los ciclos de refinamiento desarrollados hasta ahora han permitido la modificación del prototipo en base a aspectos formales, extrayéndose las siguientes conclusiones preliminares:

- La importancia de adoptar un enfoque guiado para que las tareas de indagación sean exitosas.

- La importancia del adoptar un enfoque explícito para la adecuada reflexión sobre la NDC y el PC.

Estas conclusiones son coherentes con las encontradas en trabajos previos, en los que se destaca el papel crucial del profesorado para lograr que las tareas de indagación sean efectivas (Abrami et al., 2015), así como el necesario carácter explícito de las tareas sobre NDC y PC para hacer visible y promover la reflexión adecuada sobre estos constructos (Khishfe y Abd-El-Khalick, 2002; Marin y Halpern, 2011). Estos aspectos justifican la necesidad de proporcionar un andamiaje al alumnado a través de cuestiones metacognitivas que le hagan reflexionar acerca de su conocimiento y la manera de dirigirlo, explicitando las estrategias de aprendizaje implicadas en la resolución satisfactoria de la tarea (Halpern, 2014), así como de destacar la importancia de los aspectos sociales y culturales que contextualizan la construcción del conocimiento científico (Acevedo et al., 2017).

Respecto a la motivación observada en el alumnado, puede asociase al contexto y tipo de tareas propuestas. Tal y como señalan Olivares, Saiz y Rivas (2013), los individuos se interesan y se esfuerzan en aprender aquello que van a poner en práctica y va a ser útil en su vida. En este caso, las tareas parecían cubrir las necesidades personales de los estudiantes, pues dotaban de funcionalidad al conocimiento científico, al permitirles explicar fenómenos de su vida diaria, promoviendo su entusiasmo e interés.

Los resultados que se obtengan de los datos recogidos en ambos ciclos de refinamiento, permitirán valorar cuantitativa y cualitativamente la efectividad del prototipo respecto a la mejora de la comprensión de la NDC y el desarrollo del PC del profesorado en formación inicial participante. Además, dichos resultados dirigirán el enfoque de la mejora del prototipo, que se prevé que sea implementado en nuevos grupos para corroborar, modificar y/o ampliar las conclusiones extraídas, así como para valorar el potencial de la estrategia de instrucción empleada, basada en la indagación y la HDC, para la consecución de nuestros objetivos.

\section{Agradecimientos}

Este trabajo forma parte del proyecto de investigación "Educación de las Competencias Científica, Tecnológica y Pensamiento Crítico Mediante la Enseñanza de Temas de 
Naturaleza de Ciencia y Tecnología" (EDU2015-64642-R) (MINECO/FEDER), con financiación del Ministerio de Economía y Competitividad de España y el Fondo Europeo de Desarrollo Regional.

\section{Referencias bibliográficas}

Abrami, P.C., Bernard, R.M., Borokhovski, E., Waddington, D.I., Wade, C.A. y Persson, T. (2015). Strategies for teaching students to think critically: a meta-analysis. Review of Educational Research, 85(2), 275-314.

Acevedo-Díaz, J.A. y García-Carmona, A. (2016). «Algo antiguo, algo nuevo, algo prestado». Tendencias sobre la naturaleza de la ciencia en la educación científica. Revista Eureka Sobre Enseñanza Y Divulgación De Las Ciencias, 13(1), 3-19. Recuperado de: http://hdl.handle.net/10498/18010

Acevedo-Díaz, J.A., García-Carmona, A. y Aragón, M.M. (2017). Historia de la ciencia para enseñar naturaleza de la ciencia: una estrategia para la formación inicial del profesorado de ciencia. Educación Química, 28(3), 140-146. DOI: https://doi. org/10.1016/j.eq.2016.12.003

Akgun, A. y Duruk, U. (2016). The investigation of preservice science teachers' critical thinking dispositions in the context of personal and social factors. Science Education International, 27(1), 3-15. Recuperado de: https://eric.ed.gov/?id=EJ1100164

Blanco-López, A., España-Ramos, E. y Franco-Mariscal, A.J. (2017). Estrategias didácticas para el desarrollo del pensamiento crítico en el aula de ciencias. Ápice. Revista de Educación Científica, 1(1), 107-115. DOI: https://doi.org/10.17979/arec.2017.1.1.2004

Demirdöğen, B., Hanuscin, D.L., Uzuntiryaki-Kondakci, E. y Köseoğlu, F. (2016). Development and nature of preservice chemistry teachers' pedagogical content knowledge for nature of science. Research in Science Education, 46(4), 575-612.

Dermihan, E. y Köklükaya, A.N. (2014). The critical thinking dispositions of prospective science teachers. Procedia-Social and Behavioral Sciences, 116, 1551-1555. DOI: https://doi.org/10.1016/j.sbspro.2014.01.433

Driver, R., Learch, J., Millar, R. y Scott, P. (1996). Young people's images of science. Philadelphia: Open University Press.

Ennis, R. (1991). Critical thinking: a streamlined conception. Teaching Philosophy, 14(1), 5-24.

Facione, P.A. (2011). Critical thinking: What it is and why it counts. Millbrae: California Academic Press. Recuperado de: www.student.uwa.edu.au/_data/assets/ pdf_file/0003/1922502/Critical-Thinking-What-it-is-and-why-it-counts.pdf

Halpern, D.F. (2014). Thought and knowledge. An Introduction to Critical Thinking. New York: Psychology Press.

Halpern, D.F. (2010). The Halpern critical thinking assessment: Manual. Vienna: Schuhfried.

Irez, S. (2006). Are we prepared? An assessment of preservice science teacher educators' beliefs about nature of science. Science Education, 90, 1113-1143. DOI: https://doi. org/10.1002/sce.20156

Jiménez-Aleixandre, M.P. y Puig, B. (2012). Argumentation, evidence evaluation and critical thinking. En B.J. Fraser, K.G. Tobin y C.J. McRobbie (Eds.), Second International Handbook of Science Education (pp. 1001-1015). Dordrecht: Springer. 
Khishfe, R. y Abd-El-Khalick, F. (2002). Influence of explicit and reflective versus implicit inquiry-oriented instruction on sixth graders' views of nature of science. Journal of Research in Science Teaching, 39(7), 551-578.

Lederman, N.G., Abd-El-Khalick, F., Bell, R.L. y Schwartz, R.S. (2002). Views of nature of science questionnaire: toward valid and meaningful assessment of learners' conceptions of nature of science. Journal of Research in Science Teaching, 39(6), 497-521.

Manassero, M.A., Vázquez, A. y Acevedo, J.A. (2001). Avaluació dels temes de ciència, tecnologia i societat. Palma de Mallorca: Conselleria d'Educació i Cultura del Govern de les Illes Ballears.

Marin, L.M. y Halpern, D.F. (2011). Pedagogy for developing critical thinking in adolescents: Explicit instruction produces greatest gains. Thinking Skills and Creativity, 6(1), 1-13.

McComas, W.F. (2011). The history of science and the future of science education. En P.V. Kokkotas, K.S. Malamitsa y A.A. Rizaki (Eds.), Adapting historical knowledge production to the classroom (pp. 37-53). Rotterdam: SensePublishers.

Moreno, L., Zúñiga, K., Cofré, H., Merino, C. (2018). Efecto (¿o no?) de la inclusión de naturaleza de la ciencia en una secuencia para el aprendizaje y la aceptación de la teoría de la evolución. Revista Eureka sobre Enseñanza y Divulgación de las Ciencias, 15(3), 3105. DOI: https://doi.org/10.25267/Rev_Eureka_ensen_divulg_ cienc.2018.v15.i3.3105

OCDE (2017). PISA 2015 Science Framework. En OCDE (Eds.), PISA 2015 Assessment and Analytical Framework: Science, Reading, Mathematic, Financial Literacy and Collaborative Problem Solving (pp. 19-46). Paris: OECD Publishing. DOI: http://doi. org/10.1787/9789264281820-3-en

Olivares, S., Saiz, C. y Rivas, S.F. (2013). Motivar para pensar críticamente. Electronic Journal of Research in Educational Psychology, 11(2), 367-394. Recuperado de: http://www.redalyc.org/html/2931/293128257004/

Qing, Z., Jing, G. y Yan, W. (2010). Promoting preservice teachers' critical thinking skills by inquiry-based chemical experiment. Procedia-Social and Behavioral Sciences, 2(2), 4597-4603. DOI: https://doi.org/10.1016/j.sbspro.2010.03.737

Romero-Ariza, M. (2014). Uniendo investigación, política y práctica educativas: DBR, desafíos y oportunidades. magis, Revista Internacional de Investigación en Educación, 7(14), 159-176. DOI: https://doi.org/10.11144/Javeriana.M7-14.UIPP

Solbes-Matarredona, J.S. y Torres-Merchán, N.Y. (2013). ¿Cuáles son las concepciones de los docentes de ciencias en formación y en ejercicio sobre el pensamiento crítico? Tecné Episteme Y Didaxis, 33, 61-85. DOI: https://doi.org/10.17227/01213814.33ted61.85

Valenzuela, J. (2007). Escala motivacional de pensamiento crítico. Salamanca: Facultad de Psicología, Universidad de Salamanca. Recuperado de: http://tinyurl.com/EMPC-sp

Valenzuela, J., Nieto, A.N. y Muñoz, C. (2014). Motivación y disposiciones: enfoques alternativos para explicar el desempeño de habilidades de pensamiento crítico. Revista Electrónica de Investigación Educativa, 16(3), 16-32. Recuperado de: http:// redie.uabc.mx/vol16no3/contenido-valnieto.html

Valenzuela, J. y Saiz, C. (2010). Percepción sobre el coste de pensar críticamente en universitarios chilenos y españoles. Electronic Journal of Research in Educational Psychology, 8(2), 689-706. DOI: https://doi.org/10.25115/ejrep.v8i21.1387 
Vázquez-Alonso, A. y Manassero-Mas, M.A. (2018). Más allá de la comprensión científica: educación científica para desarrollar el pensamiento. Revista Electrónica de Enseñanza de las Ciencias, 17(2), 309-336. Recuperado de: http://reec.uvigo.es/ volumenes/volumen17/REEC_17_2_02_ex1065.pdf

Vázquez-Alonso, A. y Manassero-Mas, M.A. (2013). La comprensión de un aspecto de la naturaleza de la ciencia y tecnología: Una experiencia innovadora para profesores en formación inicial. Revista Eureka sobre Enseñanza y Divulgación de las Ciencias, 10(Num. Extraordinario), 630-648. Recuperado de: https://revistas.uca.es/index. php/eureka/article/view/2813

Yacoubian, H.A. (2015). A framework for guiding future citizens to think critically about nature of science and socioscientific issues. Canadian Journal of Science, Mathematics and Technology Education, 15(3), 248-260.

Yacoubian, H.A. y BouJaoude, S. (2010). The effect of reflective discussions following inquiry-based laboratory activities on students' views of nature of science. Journal of Research in Science Teaching, 47(10), 1229-1252.

Zoller, U. y Nahum, T.L. (2012) From teaching to KNOW to learning to THINK in science education. En B.J. Fraser, K.G. Tobin y C.J. McRobbie (Eds.), Second International Handbook of Science Education (pp. 209-229). Dordrecht: Springer. 